\title{
Thymic epithelial progenitor cells and thymus regeneration: an update
}

\author{
Lianjun Zhang ${ }^{1,2}$, Liguang Sun ${ }^{1,3}$, Yong Zhao ${ }^{1}$ \\ ${ }^{I}$ Transplantation Biology Research Division, State Key Laboratory of Biomembrane and Membrane Biotechnology, Institute of Zo- \\ ology, ${ }^{2}$ Graduate University of the Chinese Academy of Sciences, Chinese Academy of Sciences, Beijing 100080, China, ${ }^{3}$ School of \\ Public Health, Jilin University, Changchun 130021, China
}

The thymus provides the essential microenvironment for T-cell development and maturation. Thymic epithelial cells (TECs), which are composed of thymic cortical epithelial cells (cTECs) and thymic medullary epithelial cells (mTECs), have been well documented to be critical for these tightly regulated processes. It has long been controversial whether the common progenitor cells of TECs could give rise to both cTECs and mTECs. Great progress has been made to characterize the common TEC progenitor cells in recent years. We herein discuss the sole origin paradigm with regard to TEC differentiation as well as these progenitor cells in thymus regeneration.

Cell Research (2007) 17:50-55. doi:10.1038/sj.cr.7310114; published online 5 December 2006

Keywords: thymic epithelial progenitor cells, thymus organogenesis, thymus regeneration

\section{Introduction}

In general, stem cells are characterized by two fundamental properties of self-renewal potential and further differentiation into several specialized cell types. Stem cells, especially tissue- or organ-specific stem cells obtained from adults, which hold great clinical potential, have attracted much attention recently [1-3]. The tissue stem cells or somatic stem cells from numerous adult tissues, with striking potential ability to repair damaged tissues and even to differentiate into other tissue and cell types, have been identified in both animals and humans [4-6]. Tissue-specific stem cells generally undergo asymmetric division, which thus gives rise to two different kinds of daughter cells. One population continues to maintain the multipotent potential whereas the secondary population becomes specialized cells responsible for the function of the tissue or organ.

It has been well defined that the thymus provides an optimal and essential microenvironment for T-cell development and maturation, although the exact molecular signal-

Correspondence: Yong Zhao

Tel: +86-10-62538391; Fax: +86-10-62659958;

E-mail: zhaoy@ioz.ac.cn ling events still remain poorly understood [7-9]. Only the thymocytes recognizing major histocompatibility complex (MHC)-self peptide complexes in an appropriate avidity can survive the stringent positive and negative selections and then become functionally and phenotypically mature $[10,11]$. This process generally involves extensive interactions between developing thymocytes and thymic stromal cells [12-17]. The thymic stromal cells may provide the growth factors, cytokines and extracellular matrix for T-cell development [18], among which thymic epithelial cells (TECs) form the basic three-dimensional architecture that has the ability to facilitate T-cell differentiation and maturation from the common lymphoid progenitors. The proper differentiation and organization of different TECs is pivotal for both thymocyte development and T-cell repertoire selection. Moreover, TECs are divided into two major categories: thymic cortical epithelial cells (cTECs) and thymic medullary epithelial cells (mTECs), which have been demonstrated to participate in positive and negative selection, respectively [12]. In addition, thymic non-epithelial stromal cells have also been suggested to play important roles at various stages of T-cell development $[17,19]$.

Homeostasis of epithelial tissues is usually maintained by continuous self-renewal of epithelial stem cells. Although it remains controversial whether the common stem 
cells for both cTECs and mTECs reside in the thymus, an emerging body of data support the existence of thymic epithelial progenitor/stem cells (TEPCs/TESCs) [20-24]. Significant progress has been made to identify and characterize TEPCs using lineage track analysis and clonal assays in recent years. In this manuscript, we will summarize data concerning the identification, characterization and purification of TEPCs as well as the potential therapeutic application of TEPCs in thymus regeneration.

\section{Thymus organogenesis}

Now, there are at least two strategies to study early thymus organogenesis in the mouse: fluorescent cell tracker and ectopic grafting technique for in vitro and in vivo analysis, respectively [25]. The former mainly focuses on characterizing the location of a distinct population of cells in the developing thymus in vitro by labelling them with a fluorescent cell tracker dye. On the other hand, ectopic grafting technique is used to test the contribution of the isolated thymic tissue in thymus organogenesis. It is commonly accepted that the thymus and parathyroids arise from a common embryonic region that develops from the third pharyngeal pouch [26]. The thymic rudiment is first visible at about embryonic day 10.5 (E10.5), and it has been suggested that all the three germ layers contribute to the thymus organogenesis. This process is generally divided into two stages: the early stage involves epithelialmesenchymal interactions in the absence of thymocytes whereas there are mutual interactions between epithelial cells and developing thymocytes at the later stage [27-32]. Epithelial-mesenchymal interactions have been implicated during organogenesis, although the initiating signals for thymus organogenesis remain elusive. It has been well documented that mesenchymal cells play critical roles at different stages of thymus organogenesis [19, 32]. Previous reports have suggested that mesenchymal cells may respond to the initiating instructive signals for thymus induction from the endoderm and in turn support the growth and differentiation of the thymic epithelial rudiment. At E10, neural crest-derived mesenchymal cells initiate physical interaction with the third pharyngeal pouch and subsequently establish the thymic primordium. At about E11, the thymic rudiment begins budding and outgrowth, followed by immigration of the lymphocyte precursors, and then the patterning and differentiation are initiated through epithelial-thymocyte interaction within the mesenchymalderived capsule [27].

Mutant or genetically modified animal models have provided valuable tools to elucidate molecular regulators responsible for thymus organogenesis. Many transcription factors have been demonstrated to be critical for thymus organogenesis and TEC differentiation. Deficiency of either the paired box family transcription factors Pax1 or Pax9 results in the failure of thymus organogenesis [33-36]. In addition, it has been reported that the Hox transcription factor family member, Hoxa3, is expressed by both the thymic epithelial and mesenchymal cells, and mutation of Hoxa3 leads to athymia [37]. The forkhead transcription factor FoxN1 is well known for the famous nude phenotype in mice, rats and humans [38, 39]. Although FoxN1 is expressed by all TECs throughout ontogeny, the TEC differentiation consists of FoxN1-independent and FoxN1dependent stages. In the absence of FoxN1, the initial steps of thymus organogenesis and the formation of the thymus 'anlage' are not impaired. However, further expansion and differentiation of the epithelial cells are prevented, which leads to non-formation of the normal three-dimensional thymic architecture and impaired ability to attract bone marrow-derived lymphoid progenitors [40]. Furthermore, Wnt proteins have been reported to induce FoxN1 expression in TECs [31, 41]. A better understanding of the earlier signalling cascades for thymus rudiment positioning and patterning [40] and detailed studies on the regulation of FoxN1 expression pattern will provide valuable information on thymus organogenesis and TEC differentiation.

\section{cTEC and mTEC differentiation: the new para- digm}

Previous morphological and histological studies have suggested the dual-origin model for thymus organogenesis, describing that the cortical epithelium derives from the surface ectoderm of the third pharyngeal cleft, whereas the medullary epithelium derives from the endoderm [29]. But this prevailing view of the dual-origin model for thymus organogenesis has been challenged recently, as both the cell marker and functional studies support the notion that cTECs and mTECs share the same endodermal origin [42-44]. The excellent evidence for the single endoderm origin is provided by chick-quail chimera experiments demonstrating that only the quail endodermal region can produce the functional mature thymus and support the normal thymopoiesis. The lineage analysis shows no evidence for the ectodermal contribution to the thymic rudiment; on the other hand, the isolated pharyngeal endoderm develops a functional thymus with well-organized cortical and medullary compartments when transplanted ectopically [45]. In this regard, the single endoderm origin of TECs is at present preferentially accepted in place of the dual-origin model [24, 29].

Chimeric mice were established by injecting embryonic stem (ES) cells into blastocysts containing the different MHC to test TEPC activity. Analysis of the 'balanced chi- 
meras', with roughly equal contributions of the ES cells and blastocyst, indicated that the medulla comprises individual epithelial 'islets', each arising from a single progenitor [46]. However, whether the cortex compartmentalization can be derived from a single progenitor cell remains to be addressed.

\section{Identification and characteristics of TEPCs}

Although the view that both cortical and medullary epithelial cells derived from the endoderm was commonly accepted, it remains controversial whether these two morphologically and functionally distinct types of TECs arise from a common bipotent progenitor. As we know, thymic stromal cells are highly heterogenous [47], and the lack of markers specific for distinct subpopulations has significantly hampered our efforts toward further identification and characterization of these cells. The development of a series of panels of monoclonal antibodies $(\mathrm{mAb})$ against different molecule determinants on distinct stromal cells provides valuable tools for further investigation on the contributions of thymic stroma to TEC development [48, 49]. Cell marker studies have demonstrated that the TEC population is homogeneous at the earlier stage of embryonic development. Cytokeratin5 and cytokeratin8 have been identified as markers of cTECs and mTECs in the adult thymus, respectively [50]. Interestingly, during the embryonic development there is a minor population of TECs coexpressing cytokeratin5 and cytokeratin8, raising the possibility of the existence of a rare progenitor cell that may give rise to both cortical and medullary TECs (Figure 1). Several lines of evidence further support the presence of the common precursors by showing that all the epithelial cells of the third pharyngeal pouch at E10.5 express both 4F1 and IVC4 which mark the cTECs and mTECs, respectively [24].

In keeping with previous studies, Gill et al. and Bennett et al. $[22,23]$ identified a special population of TECs recognized by the MTS24 and MTS20 mAbs. The tiny population of the MTS20 $0^{+}$TSS $24^{+}$cells expresses both cytokeratin5 and cytokeratin8, consistent with the existence of the potential common progenitors for cTECs and mTECs. Moreover, the putative progenitor marker MTS24 is expressed by almost all cytokeratin-positive cells at E12 [21]. To our excitement, the MTS20 $0^{+}$MTS24 ${ }^{+}$ cell population supports the development of both the cortical and medullary compartment characterized by several markers specific for cTECs and mTECs, respectively. Furthermore, they can establish a functional thymus and support T-cell development and maturation when they are re-aggregated in vitro for $24 \mathrm{~h}$ and are transplanted into the kidney capsule of nude mice, even by using as

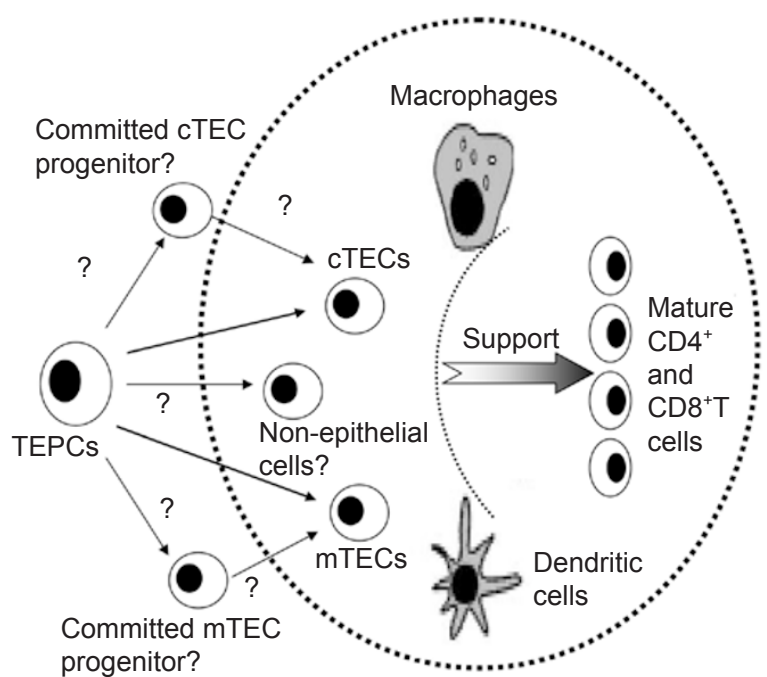

Figure 1 Schematic overview of the precursor-progeny relationship of TECs. The bipotent TEPCs do exist in both fetal and postnatal period, which then give rise to cTECs and mTECs. The TEPCs coexpress markers, which are restricted to adult cTECs and mTECs, respectively. But it remains unclear whether the progenitor cells differentiate into cTECs and mTECs directly or indirectly. cTECs and mTECs, in combination with macrophages, dendritic cells and some other non-epithelial cells, set up the appropriate microenvironment for T-cell development and maturation.

few as $500 \mathrm{MTS}^{2} 0^{+} \mathrm{MTS} 24^{+}$cells $[22,23]$. In contrast, the corresponding MTS20-MTS24 population could not support the apparent cTEC and mTEC development and the peripheral T-cell reconstitution when transplanted in the same manner. Eighty percent of MTS20-MTS24 cells express cytokeratin 5 and $10 \%$ express cytokeratin8, indicating that some differentiated mTECs and cTECs exist, but it is still unclear why those differentiated cells cannot continue to support T-cell development [23]. Although these studies provide compelling evidence that the potential TEPCs are restricted to the MTS20 $0^{+} \mathrm{MTS}_{2} 4^{+}$cells and that the implantation of MTS20 $0^{+} \mathrm{MTS}_{2} 4^{+}$cells can generate a functional microenvironment for T-cell development, the MTS20 ${ }^{+} \mathrm{MTS}_{24} 4^{+}$population is heterogenous so that it may contain several types of cells, and thus it remains obscure whether a single stem cell can fulfill functional reconstitution of the thymus. Clonal assay provides the appropriate model to define the differentiation potential of a single cell. Recently, Rossi et al. [21] provided direct evidence that a single progenitor cell can give rise to both cTECs and mTECs as defined by enhanced yellow fluorescent protein (eYFP) expression. Ideal clonal analysis requires a normal microenvironment, which can provide developmental cues 
for the stem cell to fulfill their differentiation potential; for this purpose, one YFP-expressing E12 MTS24 $4^{+}$progenitor cell was microinjected into the syngeneic wild-type thymic rudiment controlling the intact three-dimensional structure and then placed under the kidney capsule [21]. Four weeks after the transplantation, YFP expression was observed in both the cortical and medullary compartment as determined by immunofluorescence analysis with several different markers specific for cTECs and mTECs [21].

Now, we know that the MTS20 $0^{+} \mathrm{MTS}_{2} 4^{+}$population has been identified at the embryonic period (either E12.5 or E15.5) [22, 23], but whether the TEPCs exist in the postnatal period remains unclear. Notably, the TEPCs of the thymus rudiment can survive into the adult period in the absence of FoxN1. Thus, using the Cre-Loxp system, Bleul et al. [20] studied the function of postnatal TEPCs. Using the well-established Rosa26R-eYFP as a read-out for Cre activity, they elegantly introduced FoxN1 into the potential TEPCs from the nude mice thymic rudiment. They found that a single epithelial progenitor cell from the FoxN1-deficient postnatal thymus when reverted to express the FoxN1, could give rise to a complete and functional thymic microenvironment, and the peripheral $\mathrm{T}$ cells were repopulated to some extent in the nude mice, suggesting that cell-based therapies could be developed for thymic disorders . However, it is still unclear whether these cells represent the true adult TEPCs. In this regard, what strikes us most may be the capacity to generate an organ from a small population of cells or even one stem cell. Is it the same case in humans? Are these bipotent TEPCs? Two recent papers published in Nature developed novel means to track mammary epithelial stem cells; and an entire functional mammary gland could be regenerated when transplanting only one stem cell ectopically [51-53]. Can we reconstitute functional T-cell development by transplanting a defined population of the TEPCs? If so, TEPC transplantation will greatly benefit older people who have severe thymic atrophy, patients following chemotherapy and radiotherapy, or those infected with the HIV virus. In other words, we may have another chance to build a functional thymus by implanting a few TEPCs [54].

Collectively, several questions regarding TEPCs and their precise role in T-cell development remain to be resolved. One key issue in stem cell therapy is to ensure that the stem cells are placed in the appropriate location to maintain their multipotency, as the niche is critically required for the maintenance of stem cell properties including proliferation and differentiation of stem cells [55-57]. The nature of the TEPC niche and how TEPCs and their supporting cells are nested together remain to be determined. Although it is increasingly obvious that TEPCs contribute to the majority of cTECs and mTECs, we cannot exclude the possibility that the lineage-committed progenitors for either cTECs or mTECs may also exist (Figure 1). Do those bipotent progenitor cells only differentiate into cTECs and mTECs? The epithelial-mesenchymal transition has been well characterized as the fundamental process in development as well as the major mechanism for tumor metastasis. It remains unclear whether these progenitors could differentiate into several non-epithelial stromal cell types including the dendritic cells and macrophages, which also contribute to T-cell development and T-cell repertoire shaping (Figure 1). However, immunohistochemical analysis of grafting experiments by Rossi et al. [21] showed no evidence for the transition of these progenitor cells to other lineages. It is also important to ensure that the transplanted tissue can give rise to a normally developed medullary compartment, which can mediate efficient negative selection and prevent the development of autoimmune diseases, while maintaining the ability to reconstitute the peripheral T-cell compartment. Being able to do so may significantly prompt the use of TEPC transplantation in thymus regeneration.

\section{Concluding remarks and perspectives}

Although it is increasingly apparent that a bipotent progenitor cell exists at both the embryonic and postnatal period, further investigations are definitely required to characterize the exact role of MTS24 antigen in the function and development of TEPCs. Are there some differences between embryonic and adult MTS24 $4^{+}$epithelial cells? While ectopic grafting, the E12.5 or E15.5 MTS24 ${ }^{+}$cells has been proposed to support functional thymus regeneration, so far there is no evidence to document the thymus reconstitution potential of the adult MTS24 $4^{+}$epithelial cells when ectopically transplanted. Thus, it still remains questionable whether MTS24 can serve as a reliable marker to characterize adult TEPCs. In addition, is MTS24 associated with the self-renewal potential or with the proliferation and differentiation of TEPCs? Nevertheless, the increasing knowledge on TEPCs raises hopes for us to achieve thymus regeneration from implanting TEPCs in the future.

\section{Acknowledgments}

We thank Mr Aqeel Javeed for his critical review of our manuscript. This work was supported by grants from the National Basic Research Program of China (973 Program, 2003CB515501), Knowledge Innovation Program of Chinese Academy of Sciences (KSCX2-SW-333), National Natural Science Foundation for Distinguished Young Scholars (C03020504), 100 Quality Vocational Colleagues of Chinese Academy of Sciences (2003-85), and the Scientific Research Foundation for the Returned 
Overseas Chinese Scholars of State Education Ministry (2005-546).

\section{References}

1 Rando TA. The adult muscle stem cell comes of age. Nat Med 2005; 11:829-831.

2 Haas S, Weidner N, Winkler J. Adult stem cell therapy in stroke. Curr Opin Neurol 2005; 18:59-64.

3 Kofman AV, Theise ND, Hussain MA. Paradigms of adult stem cell therapy for type 1 diabetes in mice. Eur J Endocrinol 2004; 150:415-419.

4 Levy YS, Stroomza M, Melamed E, Offen D. Embryonic and adult stem cells as a source for cell therapy in Parkinson's disease. J Mol Neurosci 2004; 24:353-386.

5 Rietze RL, Valcanis H, Brooker GF, Thomas T, Voss AK, Bartlett $\mathrm{PF}$. Purification of a pluripotent neural stem cell from the adult mouse brain. Nature 2001; 412:736-739.

6 Johansson CB, Momma S, Clarke DL, Risling M, Lendahl U, Frisen J. Identification of a neural stem cell in the adult mammalian central nervous system. Cell 1999; 96:25-34.

7 Guidos C. Thymus and T-lymphocyte development: what is new in the 21 st century? Immunol Rev 2006; 209:5-9.

8 Mohtashami M, Zuniga-Pflucker JC. Three-dimensional architecture of the thymus is required to maintain delta-like expression necessary for inducing T cell development. J Immunol 2006; 176:730-734.

9 Anderson G, Harman BC, Hare KJ, Jenkinson EJ. Microenvironmental regulation of $\mathrm{T}$ cell development in the thymus. Semin Immunol 2000; 12:457-464.

10 Anderson G, Hare KJ, Jenkinson EJ. Positive selection of thymocytes: the long and winding road. Immunol Today 1999; 20:463-468.

11 von Boehmer H, Kisielow P. Self-nonself discrimination by T cells. Science 1990; 248:1369-1373.

12 Takahama Y. Journey through the thymus: stromal guides for T-cell development and selection. Nat Rev Immunol 2006; 6:127-135.

13 Hollander G, Gill J, Zuklys S, Iwanami N, Liu C, Takahama Y. Cellular and molecular events during early thymus development. Immunol Rev 2006; 209:28-46.

14 Gill J, Malin M, Sutherland J, Gray D, Hollander G, Boyd R. Thymic generation and regeneration. Immunol Rev 2003; 195:28-50.

15 Bhakta NR, Lewis RS. Real-time measurement of signaling and motility during $\mathrm{T}$ cell development in the thymus. Semin Immunol 2005; 17:411-420.

16 Anderson G, Jenkinson EJ. Lymphostromal interactions in thymic development and function. Nat Rev Immunol 2001; 1:31-40.

17 Anderson G, Jenkinson EJ, Moore NC, Owen JJ. MHC class II-positive epithelium and mesenchyme cells are both required for T-cell development in the thymus. Nature 1993; 362:70-73.

18 Yarilin AA, Belyakov IM. Cytokines in the thymus: production and biological effects. Curr Med Chem 2004; 11:447-464.

19 Suniara RK, Jenkinson EJ, Owen JJ. An essential role for thymic mesenchyme in early T cell development. J Exp Med 2000; 191:1051-1056.

20 Bleul CC, Corbeaux T, Reuter A, Fisch P, Monting JS, Boehm T. Formation of a functional thymus initiated by a postnatal epithelial progenitor cell. Nature 2006; 441:992-996.

21 Rossi SW, Jenkinson WE, Anderson G, Jenkinson EJ. Clonal analysis reveals a common progenitor for thymic cortical and medullary epithelium. Nature 2006; 441:988-991.

22 Gill J, Malin M, Hollander GA, Boyd R. Generation of a complete thymic microenvironment by MTS24(+) thymic epithelial cells. Nat Immunol 2002; 3:635-642.

23 Bennett AR, Farley A, Blair NF, Gordon J, Sharp L, Blackburn CC. Identification and characterization of thymic epithelial progenitor cells. Immunity 2002; 16:803-814.

24 Blackburn CC, Manley NR, Palmer DB, Boyd RL, Anderson G, Ritter MA. One for all and all for one: thymic epithelial stem cells and regeneration. Trends Immunol 2002; 23:391-395.

25 Gordon J, Wilson VA, Moore-Scott BA, Manley NR, Blackburn CC. In vivo and in vitro assays of thymic organogenesis. Methods Mol Med 2005; 105:303-310.

26 Gordon J, Bennett AR, Blackburn CC, Manley NR. Gcm2 and Foxn1 mark early parathyroid- and thymus-specific domains in the developing third pharyngeal pouch. Mech Dev 2001; 103:141-143.

27 Nehls M, Kyewski B, Messerle M, Waldschutz R, Schuddekopf $\mathrm{K}$, Smith AJ, Boehm T. Two genetically separable steps in the differentiation of thymic epithelium. Science 1996; 272:886889.

28 Manley NR. Thymus organogenesis and molecular mechanisms of thymic epithelial cell differentiation. Semin Immunol 2000; 12:421-428

29 Blackburn CC, Manley NR. Developing a new paradigm for thymus organogenesis. Nat Rev Immunol 2004; 4:278-289.

30 Boehm T, Scheu S, Pfeffer K, Bleul CC. Thymic medullary epithelial cell differentiation, thymocyte emigration, and the control of autoimmunity require lympho-epithelial cross talk via LTbetaR. J Exp Med 2003; 198:757-769.

31 Pongracz J, Hare K, Harman B, Anderson G, Jenkinson EJ. Thymic epithelial cells provide WNT signals to developing thymocytes. Eur J Immunol 2003; 33:1949-1956.

32 Owen JJ, McLoughlin DE, Suniara RK, Jenkinson EJ. The role of mesenchyme in thymus development. Curr Top Microbiol Immunol 2000; 251:133-137.

$33 \mathrm{Su}$ D, Ellis S, Napier A, Lee K, Manley NR. Hoxa3 and pax1 regulate epithelial cell death and proliferation during thymus and parathyroid organogenesis. Dev Biol 2001; 236:316-329.

34 Wallin J, Eibel H, Neubuser A, Wilting J, Koseki H, Balling R. Pax1 is expressed during development of the thymus epithelium and is required for normal T-cell maturation. Development 1996; 122:23-30.

35 Mansouri A, Goudreau G, Gruss P. Pax genes and their role in organogenesis. Cancer Res 1999; 59:1707s-1709s; discussion 1709s-1710s.

36 Hetzer-Egger C, Schorpp M, Haas-Assenbaum A, Balling R, Peters H, Boehm T. Thymopoiesis requires Pax9 function in thymic epithelial cells. Eur J Immunol 2002; 32:1175-1181.

37 Manley NR, Capecchi MR. The role of Hoxa-3 in mouse thymus and thyroid development. Development 1995; 121:1989-2003.

38 Su DM, Navarre S, Oh WJ, Condie BG, Manley NR. A domain of Foxn1 required for crosstalk-dependent thymic epithelial cell differentiation. Nat Immunol 2003; 4:1128-1135.

39 Cunliffe VT, Furley AJ, Keenan D. Complete rescue of the nude mutant phenotype by a wild-type Foxn1 transgene. Mamm Ge- 
nome 2002; 13:245-252.

40 Blackburn CC, Augustine CL, Li R, et al.. The $n u$ gene acts cell-autonomously and is required for differentiation of thymic epithelial progenitors. Proc Natl Acad Sci USA 1996; 93:57425746.

41 Balciunaite G, Keller MP, Balciunaite E, et al.. Wnt glycoproteins regulate the expression of FoxN1, the gene defective in nude mice. Nat Immunol 2002; 3:1102-1108.

42 Ritter MA, Boyd RL. Development in the thymus: it takes two to tango. Immunol Today 1993; 14:462-469.

43 Ropke C, Van Soest P, Platenburg PP, Van Ewijk W. A common stem cell for murine cortical and medullary thymic epithelial cells? Dev Immunol 1995; 4:149-156.

44 Ritter MA, Palmer DB. The human thymic microenvironment: new approaches to functional analysis. Semin Immunol 1999; 11:13-21.

45 Gordon J, Wilson VA, Blair NF, et al.. Functional evidence for a single endodermal origin for the thymic epithelium. Nat Immunol 2004; 5:546-553.

46 Rodewald HR, Paul S, Haller C, Bluethmann H, Blum C. Thymus medulla consisting of epithelial islets each derived from a single progenitor. Nature 2001; 414:763-768.

47 Webb SR, Sprent J. Tolerogenicity of thymic epithelium. Eur J Immunol 1990; 20:2525-2528.

48 Takacs L, Marinova T. The ontogeny of human thymic epithelium specific antigens as defined by monoclonal antibodies. Thymus 1990; 15:147-152.

49 Godfrey DI, Izon DJ, Tucek CL, Wilson TJ, Boyd RL. The phenotypic heterogeneity of mouse thymic stromal cells. Immunology 1990; 70:66-74.

50 Klug DB, Carter C, Crouch E, Roop D, Conti CJ, Richie ER. Interdependence of cortical thymic epithelial cell differentiation and T-lineage commitment. Proc Natl Acad Sci USA 1998; 95:11822-11827.

51 Kiermer V. Single-cell breast implants. Nat Methods 2006; 3:156.

52 Shackleton M, Vaillant F, Simpson KJ, et al.. Generation of a functional mammary gland from a single stem cell. Nature 2006; 439:84-88.

53 Stingl J, Eirew P, Ricketson I, et al.. Purification and unique properties of mammary epithelial stem cells. Nature 2006; 439:993-997.

54 Terszowski G, Muller SM, Bleul CC, et al.. Evidence for a functional second thymus in mice. Science 2006; 312:284-287.

55 Li L, Xie T. Stem cell niche: structure and function. Annu Rev Cell Dev Biol 2005; 21:605-631.

56 Ohlstein B, Kai T, Decotto E, Spradling A. The stem cell niche: theme and variations. Curr Opin Cell Biol 2004; 16:693-699.

57 Spradling A, Drummond-Barbosa D, Kai T. Stem cells find their niche. Nature 2001; 414:98-104. 